\title{
Grant Report \\ The Role of Social Reward and Corticostriatal Connectivity in Substance Use ${ }^{\dagger}$
}

\author{
Daniel Sazhin ${ }^{1}$, Angelique M. Frazier ${ }^{1}$, Caleb R. Haynes ${ }^{1}$, \\ Camille R. Johnston ${ }^{1}$, Iris Ka-Yi Chat ${ }^{1}$, Jeffrey B. Dennison ${ }^{1}$, Corinne P. Bart ${ }^{1}$, \\ Michael E. McCloskey ${ }^{1}$, Jason M. Chein ${ }^{1}$, Dominic S. Fareri ${ }^{2}$, Lauren B. Alloy ${ }^{1}$, \\ Johanna M. Jarcho ${ }^{1}$, David V. Smith ${ }^{1, *}$ \\ 1 Department of Psychology, Temple University, Philadelphia, PA 19122, USA \\ 2 Gordon F. Derner School of Psychology, Adelphi University, Garden City, NY \\ 11530, USA \\ $\dagger$ This research was funded by the National Institutes of Health (R03DA046733). \\ * Correspondence: David V. Smith, Email: david.v.smith@temple.edu; \\ Tel.: +1-215-204-1552.
}

\begin{abstract}
This report describes an ongoing R03 grant that explores the links between trait reward sensitivity, substance use, and neural responses to social and nonsocial reward. Although previous research has shown that trait reward sensitivity and neural responses to reward are linked to substance use, whether this relationship is impacted by how people process social stimuli remains unclear. We are investigating these questions via a neuroimaging study with college-aged participants, using individual difference measures that examine the relation between substance use, social context, and trait reward sensitivity with tasks that measure reward anticipation, strategic behavior, social reward consumption, and the influence of social context on reward processing. We predict that substance use will be tied to distinct patterns of striatal dysfunction. Specifically, reward hyposensitive individuals will exhibit blunted striatal responses to social and non-social reward and enhanced connectivity with the orbitofrontal cortex; in contrast, reward hypersensitive individuals will exhibit enhanced striatal responses to social and non-social reward and blunted connectivity with the orbitofrontal cortex. We also will examine the relation between self-reported reward sensitivity, substance use, and striatal responses to social reward and social context. We predict that individuals reporting the highest levels of substance use will show exaggerated striatal responses to social reward and social context, independent of self-reported reward sensitivity. Examining corticostriatal responses to reward processing will help characterize the relation between reward sensitivity, social context and substance use while providing a foundation for understanding risk factors and isolating neurocognitive mechanisms that may be targeted to increase the efficacy of interventions.
\end{abstract}

\section{G Open Access}

Received: 27 May 2020

Accepted: 27 October 2020

Published: 29 October 2020

Copyright (C) 2020 by the author(s). Licensee Hapres, London, United Kingdom. This is an open access article distributed under the terms and conditions of Creative Commons Attribution 4.0 International License.
KEYWORDS: corticostriatal connectivity; neuroimaging; social reward; addiction; reward sensitivity; striatum; prospective risk 


\section{INTRODUCTION}

Substance Use Disorder (SUD) constitutes a major public health crisis. In 2018, it was estimated that 20.3 million people in the United States aged 12 years or older had a SUD in the preceding year [1]. Substance use can lead to maladaptive behavior and profound physiological and psychological changes [2,3]. Substance use is also highly comorbid with other disorders such as Major Depressive Disorder (MDD) [4]. One common observation among individuals with SUD or MDD is that they have abnormalities in reward processing [5]. People who are hyposensitive to reward may be predisposed to seek drugs to compensate and up-regulate their reward systems. However, people with a hypersensitive reward system tend to be more impulsive and risk-seeking, factors that may also contribute to substance use. These seemingly conflicting observations raise the possibility that disparate mechanisms may lead to a common clinical outcome [6,7].

Limits in our understanding of the relation between aberrant reward processing and SUD may be partially linked to the fact that reward sensitivity is often assessed using self-report measures, which may be insensitive to subtle patterns of pathophysiology. Moreover, other factors may mediate or modulate the relationship between reward sensitivity and SUD. Recent studies suggest that the pathophysiology underlying addiction may be tied to reactivity of and connectivity between the striatum and orbitofrontal cortex (OFC) [8]. Substance use may also be moderated by large-scale neural networks, including the DMN (Default Mode Network) [9-13]. Importantly, corticostriatal function is highly sensitive to social context (i.e., the presence of a peer) [14,15], and the social environment plays a critical and complex role in the etiology of SUD. Next, since OFC responses have been associated with social information [16,17], it may play an important role mediating or modulating corticostriatal connectivity. OFC connectivity with frontostriatal regions may affect striatal responses to reward [18], with altered striatal connectivity and putamen OFC connectivity in chronic ketamine users being associated with impulsivity and duration of ketamine use [19]. Despite this overlap, addiction neuroscience has rarely tested relations between social context and reward processing [20]. Moreover, individual differences and personality factors such as impulsivity and emotional intelligence may affect how people process social situations and may mediate substance use behavior.

To investigate these issues, we were awarded a National Institutes of Health (NIH) R03 grant that will allow us to conduct a pilot study that quantifies how reward-dependent corticostriatal connectivity, striatal responses to social reward, and social context relate to substance use (Figure 1). We believe that responses to social reward and social context may serve as key, understudied factors in SUD-ones that shape our understanding of how hypersensitivity and hyposensitivity to reward predict risk of SUD. At this stage, we have submitted pre-registrations for 
every experiment proposed in the grant, started data collection, and initiated preliminary data analysis. In this grant report, we describe the significance and background of this project, our specific aims, methods, anticipated results, and the implications of this research.

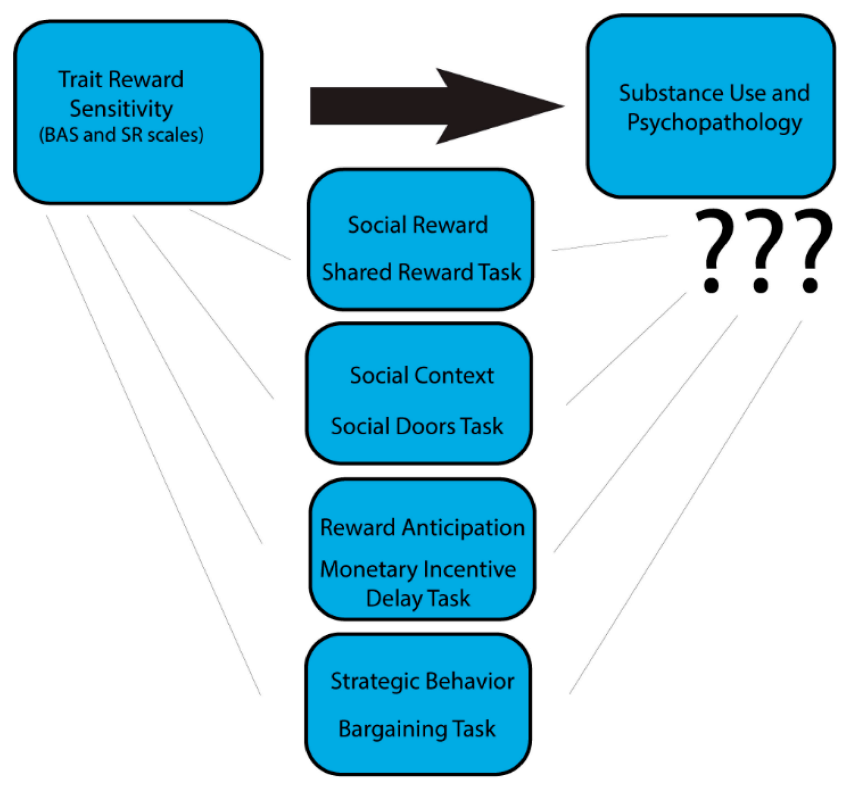

Figure 1. Relationship between Trait Reward Sensitivity and Substance Abuse and Psychopathology.We are exploring the relationship of trait reward sensitivity with substance abuse and psychopathology. Constructs that may mediate or modulate this relationship include social reward, social context, reward anticipation, and strategic behavior. Although trait reward sensitivity has been associated with substance abuse and psychopathology, it remains unclear whether this relationship is mediated and/or moderated by responses to social reward, social context, reward anticipation, and strategic behavior (middle). We evaluated these constructs in an fMRI experiment by completing an anatomical scan, Monetary Incentive Delay task, Shared Reward task, Social Doors Images and Faces respectively, the Ultimatum and Dictator Games (see main text for details about the data collection protocol). Our R03 grant will allow us to quantify how reward-dependent corticostriatal connectivity contributes to substance use and whether striatal responses to social reward and social context contribute to substance use. Piecing apart the neural mechanisms underlying reward and their relations to reward sensitivity and other individual differences will provide a broad understanding of the association of reward processing, substance abuse, and psychopathology.

\section{SIGNIFICANCE}

\section{Reward Hyposensitivity Model}

One of the leading theories of addiction is the Reward Deficiency Model of Addiction. This model proposes that addictive substances evoke activation in brain regions that receive dopaminergic inputs, particularly the striatum and ventromedial prefrontal cortex [21,22]. Repeated use over time blunts activation, leading to a diminution of engagement associated with reward [23,24]. There are two ways in which the reward circuit can be downregulated. Individuals with insufficient DA receptors can develop abnormalities in their neural reward system that predispose them to substance use [25]. Alternately, reward hyposensitivity can arise due to substance use, which downregulates the reward system in the 
absence of the substance [26]. This may lead to a repeated and compulsive behavior due to enhanced sensitivity of memory circuits to drug cues [26]. Subsequently, the behavior devolves into compulsive drug use as circuits associated with inhibition are co-opted and weakened [27]. Finally, exposure to drug cues leads to hyperactivation of motivation circuitry towards drugs, resulting in addiction [28]. Paradoxically, initial hyposensitivity to reward leads to changes in reward circuitry that become more hyperactive with continued substance use.

Studies have shown that neural hyposensitivity to reward is associated with substance use. A meta-analysis of fMRI research assessing the relation between reward processing and addiction showed that the ventral striatum of individuals with a SUD exhibited hypoactivation during reward anticipation, but hyperactivation during reward outcome [29]. Moreover, a longitudinal study with novelty-seeking adolescents showed that blunted ventral striatal responses to anticipated rewards predicted drug use [30]. Finally, in young adults, blunted reward sensitivity and trait disinhibition interacted to predict substance use problems [31]. These recent studies provide evidence that suppressed reward sensitivity is associated with substance use.

Major Depressive Disorder (MDD) is highly comorbid with substance use [32], suggesting that there may be parallels in reward processing deficiencies that affect both disorders. One third of individuals diagnosed with MDD also are diagnosed with SUDs [4]. Thus, investigating reward processing in MDD may offer additional insight into how dysregulated reward networks may also contribute to substance use. Participants with MDD exhibit attenuated functional connectivity between the VS and cortical regions, such as the vmPFC and OFC [33,34]. A meta-analysis of fMRI research assessing reward processing in MDD revealed distinct abnormalities within reward circuitry, such as a hyporesponsive ventral striatum and hyperresponsive OFC [35]. This abnormalities may lead to impaired reward prediction error encoding and a dysregulated reward system [36]. Furthermore, specific depressive symptoms (i.e., anhedonia) have been associated with diminished reward sensitivity among firstdegree family members of patients diagnosed with MDD [37], suggesting a possible genetic link between MDD and reward hyposensitivity. Together, this evidence provides support for the notion that attenuated responses to reward are associated with both substance use and with disorders that commonly co-occur with SUDs, problems that afflict millions of Americans.

\section{Reward Hypersensitivity Model}

The Reward Hypersensitivity Model proposes that individuals with higher reward sensitivity are more likely to engage in behavior that has pleasurable, yet risky consequences, including substance use. This model is an outgrowth of Gray's reinforcement sensitivity theory [38], which theorizes that there are two systems that guide motivated behavior in 
response to rewards: an approach and avoidance system. The approach system is associated with novelty-seeking, impulsivity, risk-taking, and otherwise goal-driven behavior [38]. The behavioral inhibition system (BIS) and the behavioral activation system (BAS) scales were developed as psychometric indices of these two systems in relation to reward and goaldriven behavior [39] and is also assessed with the Sensitivity to Reward scale [40]. Elevated BAS scores (i.e., hypersensitivity to reward) have been correlated with substance use, bipolar disorder [41], and impulsivity [42]. Behavioral responsivity to appetitive stimuli has been associated with neural activity in the left orbitofrontal cortex, left insula, and right ventral striatum [43] and the dorsomedial striatum [44]. Substance Dependent Individuals (SDI) had higher BAS fun seeking scores, lower decision making performance, and elevated left OFC activity, indicating this region may play a role in reward-related decision making in SUD [45]. Taken together, these studies indicate that dysregulated reward processing can then result in faulty decision making such as substance use.

Studies have found that hypersensitivity to reward is also associated with substance use. High BAS scores are associated with alcohol use [46], cocaine drug addiction [47] and nicotine dependence [48]. Adolescents with higher BAS scores are more likely to have substance use disorders [49]. Finally, mood disorders, such as bipolar spectrum disorders, are associated with substance use, impulsivity, and an elevated response to reward [41]. These findings suggest that the reward hypersensitivity model gives insight into some of the mechanisms underlying SUD.

\section{Social Reward and Social Decision Making}

A wide range of rewards, including drugs of abuse, are sought and experienced in social contexts, including sharing with peers. Moreover, negative social events, such as peer rejection, often trigger relapse [50]. Further, recent research indicates that corticostriatal connectivity to social regions such as the OFC may be associated with substance use [19,51]. Despite these observations, little is known about how striatal responses to social reward (e.g., peer rejection/acceptance) and social context (e.g., sharing a reward with a peer) are linked to reward sensitivity and substance use.

A growing body of literature suggests that the striatum plays a crucial role in processing social information. For example, studies of inequity [52], and social comparison $[53,54]$ have found that the striatum has a key role in integrating social information. The striatum may accomplish this by integrating social information through coding social context and rewards [55]. For example, higher VS activation is associated with self-disclosure [56], coding social learning signals and reciprocation from a close friend in a trust task [57]. Additionally, elevated vs activity was related to positive feedback and integrating the social context of receiving such feedback [58]. Finally, dysregulated striatal responses to social information 
processing have been implicated in symptoms of poor social competence, which are common in SUD.

Our real-world choices are often influenced by social information, which requires us to evaluate thoughts and intentions of other individuals $[59,60]$. These processes engage a network of brain regions, including the temporal-parietal junction (TPJ), the posterior cingulate cortex (PCC), and the medial prefrontal cortex (mPFC). This constellation of brain regions has been labeled the "social brain" and overlaps anatomically with the DMN [61]. Neural activation can also be biased by social context. For example, striatal reward value signals are enhanced in the presence of a peer $[17,62,63]$. This heightened engagement is linked with greater risktaking behavior, which, in turn, increased the likelihood of maladaptive consequences $[14,64]$. Although social context can influence the way we respond to nonsocial rewards (e.g., money), the reward system is also independently responsive to social reward outcomes, such as social acceptance $[65,66]$. Social comparison, such as when there are differences in payoffs between pairs of participants simultaneously completing a simple estimation task, is associated with variation in VS activation [54]. Finally, receipt of social reward has been shown to modulate connectivity between regions comprising reward circuitry (e.g., striatum and VMPFC) and social brain regions such as the TPJ [67,68] and OFC [18]. Substance use may affect corticostriatal connectivity between these social regions, with findings indicating altered striatal connectivity and putamen OFC connectivity in chronic ketamine users [19] and problematic substance use in psychiatric patients [51].

Despite these observations, very little is known about how responses to social reward and social context are linked to substance use, where social factors frequently play a critical role in consumption behavior [20,69]. For example, drugs of abuse are often consumed in social settings (e.g., binge drinking with friends), and negative social experiences (e.g., loss of a relationship or social isolation) can trigger relapse among individuals recovering from SUD [50]. These observations suggest that substance use may depend, in part, on how individuals respond to social experiences, including social reward itself, as well as social contexts associated with consumptive behavior. While social context and processing of social rewards likely contribute to substance use, it remains unclear how striatal responses to social rewards are associated with reward sensitivity and substance use, respectively.

Substance use may impact the engagement of brain regions associated with social decision making, further contributing to maladaptive or suboptimal behavior among individuals with SUD. Social decision making often requires meta-cognition-an understanding of how one's own choices can affect others. When bargaining with others, for example, people must often balance a desire to maximize their own earnings with the possibility of their offer being rejected by their partner. One underexplored factor that may increase a participant's earnings in a 
bargaining situation could be emotional intelligence, which is related to self-interested behavior in social contexts [70,71]. Other factors that enhance a participant's bargaining effectiveness included greater reward sensitivity [72], or higher levels of executive function [73], which may serve to moderate impulsivity. Another perspective as to what may drive bargaining behavior includes a participant's attitudes toward punishment and norm compliance; factors which have been associated with activations in the lateral OFC and right dorsolateral prefrontal cortex (dlPFC) [74]. However, this explanation is confounded with the possibility that subjects are motivated through maximizing their earnings rather than desiring to minimize the possibility of punishment. Little is known about how psychological factors such as emotional intelligence and impulsivity and their respective neural correlates interact to maximize earnings in bargaining situations. In summary, social incentive processing components such as strategic behavior, reward anticipation, social reward, and social context could inform how addiction impairs decision making. Although substances are often consumed in social contexts, it remains underexplored how these variables are associated with SUD. Further while reward sensitivity has been associated with substance abuse and psychopathology, it remains unclear whether this relationship is mediated or moderated through these social variables (see Figure 1).

\section{SPECIFIC AIMS}

Hyposensitivity and hypersensitivity to reward both have been implicated in substance use behavior. Recent studies suggest that the pathophysiology underlying addiction may be tied to reactivity of and connectivity between the striatum and orbitofrontal cortex (OFC) [8]. The way reward sensitivity may mediate or modulate these neural relations remains unknown. In addition, corticostriatal responses to reward are highly sensitive to social context (i.e., the presence of a peer) [14,15]. Despite this, addiction neuroscience rarely has tested the relations between social context and reward processing [20]. It is critical to address this gap given that a wide range of rewards, including drugs of abuse, are sought and experienced in social contexts, including sharing with peers.

To investigate these relations, we are assessing individual differences in reward sensitivity in a healthy college-aged sample using the Behavioral Inhibition System/Behavioral Activation System (BIS/BAS) Scales [39] and Sensitivity to Punishment (SP)/Sensitivity to Reward (SR) Questionnaire (SPSRQ) [40] in conjunction with task-based fMRI to probe responses to social and nonsocial reward. Our project is addressing two aims through exploring the relationship of trait reward sensitivity, substance use, and social context (see Figure 1).

Our first aim is to quantify how reward-dependent corticostriatal connectivity contributes to substance use. Although prior studies have established that aberrant responses on the BAS and SR are associated with substance use [41,46], it remains unclear whether reward-dependent 
corticostriatal connectivity explains additional variance in substance use. To investigate this issue, healthy young adults (ages 18-22) who vary in their reward sensitivity (as assessed by BAS and SR) and substance use patterns (as assessed by clinical interviews) will engage in wellcharacterized tasks that probe reward consumption [75] and reward anticipation [76]. We hypothesize that the degree of substance use will be greater in individuals who are either hyposensitive or hypersensitive to reward. We also hypothesize that reward hyposensitive individuals will exhibit blunted striatal responses to reward and enhanced striatal-OFC connectivity. Reward hypersensitive individuals will exhibit enhanced striatal responses to reward and blunted striatal-OFC connectivity.

Our second aim is to quantify whether striatal responses to social reward and social context contribute to substance use. Drugs of abuse are often tied to social interactions [69]; and negative social events (e.g., romantic rejection) can trigger relapse in individuals with substance use disorders [50]. Despite these links between social processes and substance use, little is known about the relations between substance use, reward sensitivity (as assessed by BAS and SR), and striatal responses to social reward and social context. To investigate this issue, our participants are engaging in tasks developed by our investigative team that probe responses to social context [62] and social reward [65,77]. We hypothesize that individuals reporting the highest levels of substance use will show exaggerated striatal responses to social reward (e.g., peer acceptance relative to rejection) and social context (i.e., sharing a monetary reward with a friend relative to a stranger), independent of self-reported reward sensitivity. In addition, we predict that elevated striatal responses to social reward/social context will be associated with enhanced connectivity with regions modulated by social information (e.g., temporal-parietal junction).

Overall, this National Institute on Drug Abuse (NIDA)-funded project will help characterize the links between substance use, reward sensitivity, and neural responses to social and nonsocial rewards. Assessing whether corticostriatal connectivity is associated with substance use would allow for a more sophisticated and nuanced understanding of the physiological mechanisms contributing to addiction. Moreover, assessing the relation between neural responses to social reward and social context and substance use could delineate social mechanisms of addiction. Understanding the psychological and physiological relations underlying substance use would inform future theoretical and experimental work in addiction research, and ultimately, could contribute to interventions and therapies for substance use disorders. 


\section{APPROACH}

\section{Grant Structure and Timeline}

In 2019, we were awarded an Imaging-Science Track Award for Research Transition (I-START) grant from the National Institute on Drug Abuse (NIDA). The I-START grant is a 1-Year R03 mechanism that is intended to support a small, proof of concept project that facilitates the transition to conducting neuroimaging studies that examine the mechanisms underlying drug use. One of the major goals of the grant is to develop pilot data that can be used in a future R01 submission to NIDA (see Summary, Limitations, and Long-Term Outlook).

To address this goal, we have assembled a study team that is led by Principal Investigator David Smith and includes four Co-Investigators from Temple University and a consultant from Adelphi University. Dr. Smith is an expert in neuroeconomics and functional neuroimaging, with extensive methodological expertise in brain connectivity analysis approaches [78,79]. Co-Investigator Lauren Alloy has over three decades of clinical experience in areas that are directly relevant to the proposal (e.g., clinical implications of aberrant reward sensitivity [6,41]. Co-Investigator Jason Chein has experience with imaging paradigms that examine the effects of social context (e.g., peers) on decision making and reward processing $[14,64]$. Co-Investigator Johanna Jarcho is an expert in social neuroscience and has substantial expertise in neuroimaging paradigms that examine social interactions, including the social/nonsocial reward paradigm used in the proposal [77]. Co-Investigator Michael McCloskey is a clinical psychologist with substantial expertise in substance use disorders [80]. Consultant Dominic Fareri is an expert in social neuroscience and pioneered the shared reward task used in the proposal [62]. Finally, we note that project coordination and data collection will be led by Angelique Frazier and Caleb Haynes. In addition, task-specific components of data analysis and dissemination will be spearheaded by Caleb Haynes (all tasks), Daniel Sazhin (Ultimatum Game; see Section Ultimatum game task), Camille Johnston (Social Doors; see Section Social doors task), Iris Ka-Yi Chat (Monetary Incentive Delay; see Section Monetary incentive delay task), Jeffrey Dennison (Monetary Incentive Delay; see Section Monetary incentive delay task), and Corinne Bart (Monetary Incentive Delay; see Section Monetary incentive delay task).

Given that data collection was active for only a few months prior to the COVID-19 outbreak, this grant report is primarily focused on providing an overview of our experiments and plans. Depending on when we can resume data collection, we hope to present preliminary results at spring conferences, such as Social and Affective Neuroscience. Following these presentations, we hope to submit manuscripts tied to each experiment. Authorship on all manuscripts will follow guidelines proposed by the International Consortium of Medical Journal Editors [81,82]. Following publication, all neuroimaging data will be made publicly available on 
OpenNeuro (https://openneuro.org) and all analysis code and stimuli will be made available on PI Smith's lab GitHub site (https://github.com/DVSLab). Each publication will also include a link to a NeuroVault.org [83] repository that contains both thresholded and unthresholded statistical maps.

\section{General Methods}

Participants, Recruitment, and Sample Characteristics. Our study is intended as a foundational project that will be extended in a subsequent R01 submission. Although we were initially aiming to collect imaging data from 100 participants between the ages of 18 and 22 years, we may have to adjust this target based on constraints imposed by the COVID-19 pandemic. Participants attended two appointments, consisting of a diagnostic interview, battery of psychometric surveys, and a mock scan, followed by a second appointment which consisted of the fMRI scan and behavioral tasks.

Participants are recruited based on scores on two self-report measures of trait reward sensitivity: BIS/BAS [39] and SPSRQ [40]. The total BAS reward scale and the Sensitivity to Reward (SR) subscale of SPSRQ are reliable and valid measures of reward sensitivity that Co-I Alloy has previously used to select participants with different levels of reward sensitivity [84,85]. Moreover, Co-I Alloy recently demonstrated that reward sensitivity, as assessed by both measures, is best characterized by dimensional, not a categorical vulnerability to bipolar disorder [86]. We are screening approximately 1000 participants on demographics and these two measures. Based on both the total BAS and SR $(r=0.40)$ scales, we will recruit participants from each quintile of reward sensitivity. In order to be included, participants must be in the same quintile on each measure of reward sensitivity. This procedure mirrors our prior work (e.g., Ref. [41]) and increases confidence that participants responded truthfully since scoring similarly on both measures would be unlikely with random or inattentive responses. If we achieve a total sample of $N=100$ across five quintiles (low to high) of reward sensitivity then each quintile will have 20 participants. This design ensures both that we will have the full dimension of reward sensitivity represented in the sample and that we will have adequate numbers of participants at risk for substance use disorders (bottom quintile and top quintile), as well as participants in the middle (control participants) at low risk for substance use. The state of recruitment prior to COVID restrictions suspended collections as of March 3rd 2020 consisted of 1472 people screened, 382 people deemed eligible, and 69, 67, 65, 75, 106 participants recruited across five quintiles (low to high) of reward sensitivity. At this stage, 236 participants completed appointment 1, 10 participants were subsequently scanned in the 1, 1, 3, 5, 0 quintiles of reward sensitivity.

Diagnostic and Symptom Measures. Participants with aberrant reward sensitivity are at increased risk for mood disorders and exhibit 
mood symptoms that could confound analyses that link substance use to brain responses to social and nonsocial reward. Thus, lifetime and family history of mood and substance use disorders based on DSM-5 criteria and Research Diagnostic Criteria (RDC) [87] will be assessed with The Structured Clinical Interview for DSM-5 (exp-SCID-5) diagnostic interview [88] by interviewers blind to participants' reward sensitivity. Complementing the mood symptom ratings in the exp-SCID-5, depression symptoms also will be assessed with the Beck Depression Inventory-II (BDI-II) [89] and the 7Down subscale of the 7Up7Down Scale (7U7D) [90]. Hypo/mania symptoms will be assessed with the Altman Self-Rating Mania Scale (ASRM) [91] and 7Up subscale of the 7U7D. These scales comprehensively assess depression and hypo/mania symptoms across a range of severity, allowing us to control for mood symptoms (via regression) and exclude participants with existing mood disorder on the exp-SCID-5.

Assessments of Substance Use. In addition to assessing DSM-5 substance use disorder criteria for alcohol and non-alcohol substances via the SCID-5, we also will administer the Timeline Follow-Back for Alcohol (TLFB-A) and non-alcohol Drugs (TLFB-D), to obtain a more nuanced assessment of current substance use frequency and patterns [92,93]. Next, we will assess substance use using the Drug Use Disorders Identification Test (DUDIT) [94], Adolescent Alcohol and Drug Involvement Scale (AADIS) [95], and the Alcohol Use Disorders Identification Test (AUDIT) [96]. These assessments have been used extensively by Co-I McCloskey, an expert in substance use disorders. For alcohol and non-alcohol substance use separately, estimates of both current substance use (TLFB) and history of problematic substance use (number of substance use criteria endorsed) will be considered in the primary analyses. However, if it is found that the two measures are collinear, TLFB and DSM-5 measures will be ztransformed and combined into a single variable. To control for the possibility that the individual may be currently under the acute effects of alcohol or illicit substance, or otherwise experiencing acute withdrawal from such a substance, we will exclude individuals with a current substance use disorder that includes symptoms of physical dependence (i.e., tolerance or withdrawal). Physical dependence is assessed through the Structured Clinical Interview for DSM. Further exclusion criteria include if a participant's self-reported AUDIT score is greater than 17 or if their response on DUDIT Q1 was greater than 3. Further, we excluded participants with current substance influence by administering a urine test and a breathalyzer test prior to behavioral and fMRI study procedures.

Social Functioning. Additional self-report measures are being administered to assess preference for social engagement and social cognitive abilities. The Adult Autism Quotient [97] assesses levels of autistic traits through items measuring constructs such as social skills, communication and attention to detail, among others. The Interpersonal Reactivity Index [98] assesses individual differences in empathy and 
mentalizing abilities via items measuring perspective taking abilities and empathic concern. The trait Emotional Intelligence (EI) questionnaire (TEIQe) measures individual differences in trait empathy, emotion regulation and perspective taking in emotional contexts [99]. The AAQ, IRI, and EI measures are being used as exploratory covariates to help clarify relations between substance use, reward sensitivity, and responses to social stimuli.

Longitudinal Effects. Since data collection has been temporarily suspended due to COVID restrictions, we have added several longitudinal measures to assess changes in substance use and anhedonia over time. We are conducting a remote followup survey administered at 6,12, and 18 months following the scan and/or initial screening. This survey assesses mood symptoms (SevenUp, SevenDown), levels of substance use (AADIS, DUDIT, AUDIT), and coping/motives through the Drinking Motives Questionnaire, Revised (DMQ-R) [100] Marijuana Motives Questionnaire (MMQ) [101], and the Distress Tolerance Scale (DTS-8) [102].

\section{Neuroimaging Methods and Analysis}

\section{Data acquisition}

Neuroimaging data are being collected at the Temple University Brain Research \& Imaging Center (TUBRIC), which houses a Siemens Prisma 3T scanner and is directed by Co-I Chein. We are acquiring $\mathrm{T}_{1}$-weighted structural images to facilitate brain normalization. Functional data are being collected using a multiband EPI sequence. See Table 1 for details regarding imaging parameters.

Table 1. Neuroimaging modalities and corresponding scanning parameters.

\begin{tabular}{l|c|c|c|c|c|c|c|c|c|c}
\hline Modality & Matrix & Slices & FOV & $\begin{array}{c}\text { \% } \\
\text { FOV } \\
\text { Phase }\end{array}$ & $\begin{array}{c}\text { Resolution } \\
\mathbf{( m m )}\end{array}$ & $\begin{array}{c}\text { TR } \\
\mathbf{( m s )}\end{array}$ & TE (ms) & $\begin{array}{c}\text { Flip } \\
\text { Angle } \\
\text { (degrees) }\end{array}$ & $\begin{array}{c}\text { Parallel } \\
\text { Imaging }\end{array}$ & $\begin{array}{c}\text { Multiband } \\
\text { Acceleration }\end{array}$ \\
\hline T1w & $224 \times$ & 192 & 224 & 100 & $1.0 \times 1.0 \times 1.0$ & 2400 & 2.17 & 8 & $2 \times$ & off \\
\hline fieldmap & $74 \times 74$ & 58 & 207 & 100 & $2.8 \times 2.8 \times 2.8$ & 645 & $\begin{array}{c}4.92 \\
\text { (GRAPPA) }\end{array}$ & 60 & off & off \\
\hline fMRI & $80 \times 80$ & 52 & 240 & 100 & $3.0 \times 3.0 \times 3.0$ & 1750 & 29 & 74 & off & 2 \\
\hline
\end{tabular}

\section{FMRI preprocessing}

At the conclusion of data collection, preprocessing will be performed using the long-term support version of fMRIPrep (20.2.0). Our preliminary analyses have utilized fMRIPrep 1.5.3 [103] which is based on Nipype 1.3.1 [104,105]. Relevant boilerplate methodological details are described below.

Anatomical data preprocessing. The T1-weighted (T1w) image will be corrected for intensity non-uniformity (INU) with N4BiasFieldCorrection 
[106], distributed with ANTs 2.2.0 [107] and used as T1w-reference throughout the workflow. The T1w-reference will then be skull-stripped with a Nipype implementation of the antsBrainExtraction.sh workflow (from ANTs), using OASIS30ANTs as target template. Brain tissue segmentation of cerebrospinal fluid (CSF), white-matter (WM) and graymatter (GM) will be performed on the brain-extracted T1w using fast [108]. Volume-based spatial normalization to two standard spaces (MNI152NLin2009cAsym, MNI152NLin6Asym) will be performed through nonlinear registration with antsRegistration (ANTs 2.2.0), using brainextracted versions of both T1w reference and the T1w template. The following templates were selected for spatial normalization: ICBM 152 Nonlinear Asymmetrical template version 2009c [109], FSL MNI ICBM 152 non-linear 6th Generation Asymmetric Average Brain Stereotaxic Registration Model [110] (RRID:SCR_002823; TemplateFlow ID: MNI152NLin6Asym).

Functional data preprocessing. For each of the BOLD runs per participant (across all tasks and sessions), the following preprocessing steps will be performed: First, a reference volume and its skull-stripped version will be generated using a custom methodology of fMRIPrep. A B0nonuniformity map (or fieldmap) will be estimated based on a phasedifference map calculated with a dual-echo GRE (gradient-recall echo) sequence, processed with a custom workflow of SDCFlows inspired by the epidewarp.fsl script and further improvements in HCP Pipelines [111]. The fieldmap then will then be co-registered to the target EPI (echo-planar imaging) reference run and converted to a displacements field map (amenable to registration tools such as ANTs) with FSL's FUGUE and other SDCflows tools. Based on the estimated susceptibility distortion, a corrected EPI (echo-planar imaging) reference will be calculated for a more accurate co-registration with the anatomical reference. Then, the BOLD reference will be co-registered to the T1w reference using FLIRT [112] with the boundary-based registration [113] cost-function. Coregistration will be configured with nine degrees of freedom to account for distortions remaining in the BOLD reference. Head-motion parameters with respect to the BOLD reference (transformation matrices, and six corresponding rotation and translation parameters) will be estimated before any spatiotemporal filtering using mcflirt [112] BOLD runs will be slice-time corrected using 3dTshift from AFNI 20160207 [114]. The BOLD time-series (including slice-timing correction when applied) will be resampled onto their original, native space by applying a single, composite transform to correct for head-motion and susceptibility distortions. These resampled BOLD time-series will be referred to as preprocessed BOLD in original space, or just preprocessed BOLD. The BOLD time-series will be resampled into several standard spaces, correspondingly generating the following spatially-normalized, preprocessed BOLD runs: MNI152NLin2009cAsym, MNI152NLin6Asym. 
Automatic removal of motion artifacts using independent component analysis [115] will be performed on the preprocessed BOLD on MNI space time-series after removal of non-steady state volumes and spatial smoothing with an isotropic, Gaussian kernel of $6 \mathrm{~mm}$ FWHM (full-width halfmaximum). In addition, several confounding time-series are calculated based on the preprocessed BOLD. Notably, three signals will be extracted within the CSF, WM, and whole-brain masks. These additional regressors add an extra layer of protection when removing residual variance associated head motion [116]. All resamplings can be performed with a single interpolation step by composing all the pertinent transformations (i.e., headmotion transform matrices, susceptibility distortion correction when available, and co-registrations to anatomical and output spaces). Gridded (volumetric) resamplings will be performed using antsApplyTransforms (ANTs), configured with Lanczos interpolation to minimize the smoothing effects of other kernels [117]. Non-gridded (surface) resamplings will be performed using mri_vol2surf (FreeSurfer).

Many internal operations of fMRIPrep use Nilearn 0.6.0 [118], mostly within the functional processing workflow. For more details of the pipeline, see the section corresponding to workflows in fMRIPrep's documentation.

\section{Neuroimaging analyses}

We will use FSL [119] to evaluate brain activation associated with each task. Each task will be modeled separately using a general linear model (GLM) with local autocorrelation correction [120]. All task-based regressors will consist of unit impulses convolved with a canonical hemodynamic response function. Each GLM will also include a set of nuisance regressors that account for non-steady state volumes, mean white matter signal, mean cerebrospinal fluid signal, and motion-related components identified with ICA-AROMA [115]. We also will include discrete cosine basis functions to filter out signals with a period exceeding $128 \mathrm{~s}$.

To measure corticostriatal interactions, we will use psychophysiological interaction (PPI) analysis [121], which estimates simple models of effective connectivity [78,122,123]. We will conduct this analysis using the ventral striatum (see Ref. [124]) as a seed. Unlike functional connectivity methods, which only measure correlations between brain regions, PPI provides a better model of neuronal coupling because PPI effects are difficult to attribute to changes in (a) another connection, (b) response levels in one region, or (c) observational noise [122,125]. These features of PPI allow us to rigorously quantify corticostriatal interactions. We will use a novel network-based PPI (nPPI) developed by PI Smith that can help clarify how large-scale networks involved in substance use (e.g., DMN) interact with other brain regions during reward processing [126,127]. Briefly, this approach extracts network-level responses (i.e., timecourses) using dual-regression analysis 
$[128,129]$ and includes those timecourses as regressors in the PPI model, thus allowing for improved quantification of effective connectivity [121].

To model data across participants, we will employ mixed-effects models [130] that assess simple main effects of each task construct (e.g., reward anticipation) and also brain-behavior correlations (e.g., reward anticipation and substance use). Our group-level models also will include nuisance regressors to account for individual differences in head motion and data quality, as we have done in our prior work [131,132]. Finally, we will correct for multiple comparisons using permutation-based analyses [133] with cluster-extent thresholding [134] and threshold-free cluster enhancement [135]. All statistical maps-both thresholded and unthresholded—will be deposited on NeuroVault [83].

\section{Experiments}

\section{Monetary incentive delay task}

Rationale. Self-report and behavioral assessments of reward sensitivity have been associated with substance use [41]. However, it remains unclear if extreme levels of aberrant behavioral reward sensitivity (i.e., hyposensitivity and hypersensitivity) and anhedonia contribute to substance use through similar or distinct neural mechanisms. To investigate this issue, we will quantify corticostriatal activation and connectivity in participants who vary in trait reward sensitivity.

Experimental Design. Participants will perform a well-established reward anticipation task called the Monetary Incentive Delay Task (MID) (Figure 2) [136]. The task will consist of 2 runs, each with 50 trials. On each trial, the participant is presented with a cue that indicates the value of the current trial $(-\$ 5,-\$ 1, \$ 0,+\$ 1$, or $+\$ 5)$, which is then followed by a target stimulus (a triangle). To earn the reward (or avoid the punishment), participants will be instructed that they must respond rapidly to the target stimulus. Neuroimaging analyses focus on the response to the cue, which isolates anticipatory responses. We will additionally examine the response to feedback (hit vs miss), which provides a measure of reward consumption.

Primary Hypotheses. (H1.1) Both hyposensitive and hypersensitive individuals will exhibit higher reported levels of substance use than individuals reporting moderate reward sensitivity. This hypothesis will be evaluated using $t$-tests comparing high/low relative to moderate and using regression analysis where reward sensitivity is fit with a quadratic function. (H1.2) Hyposensitive individuals will exhibit blunted striatal responses to reward consumption and anticipation and enhanced corticostriatal connectivity with OFC and the DMN. Hypersensitive individuals will exhibit enhanced striatal responses to reward consumption and anticipation and blunted corticostriatal connectivity with OFC and the DMN. (H2) Increased severity of anhedonia will be 
associated with blunted differences in relative motivation, defined as the difference in reaction time (RT) for large relative to small incentive cues (irrespective of reward and loss). (H3.1) Reward-hyposensitive individuals will exhibit enhanced corticostriatal connectivity with the orbitofrontal cortex (OFC) and the Default Mode Network (DMN) compared to individuals with moderate sensitivity. (H3.2) Reward-hypersensitive individuals will exhibit blunted corticostriatal connectivity with OFC and the DMN.

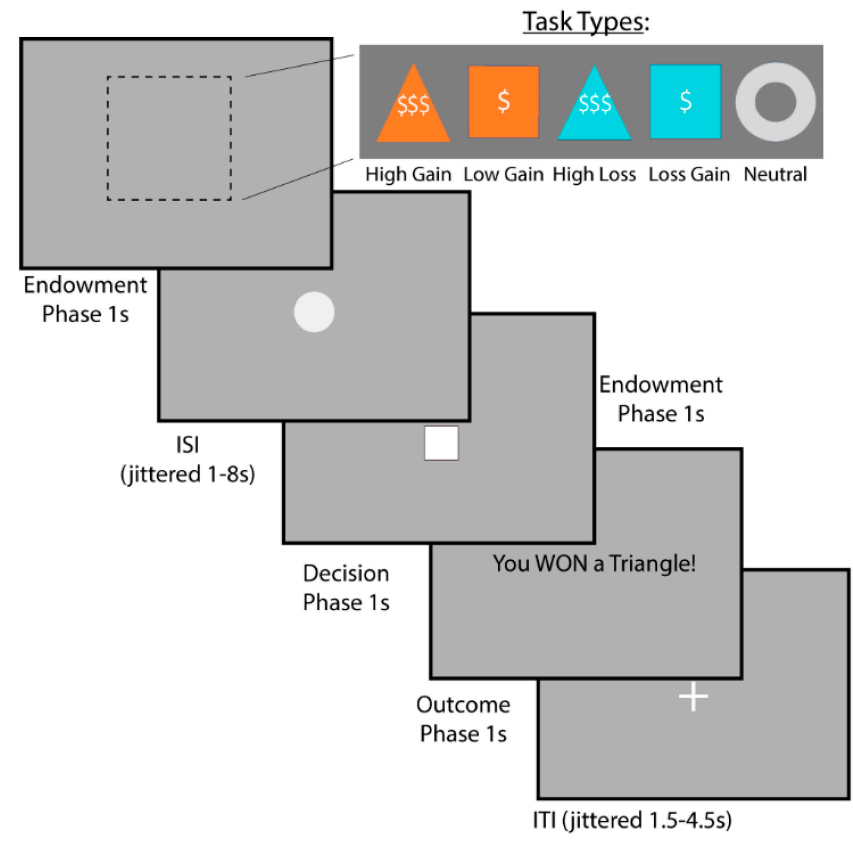

Figure 2. Measuring Reward Anticipation Using the Monetary Incentive Delay Task. To measure reward anticipation, we will use the monetary incentive delay task. This task requires the subject to respond to a stimulus as quickly as possible to win or avoid losing money. Gain trials are represented as orange, whereas loss trials are blue. Triangles represent large amounts, whereas squares small amounts. The gray circle represents neither gains or losses.

Limitations and Future Directions. Although the behavior measured with the reward anticipation is limited to reaction time, cue-dependent differences in reaction time can provide a reliable index of relative motivation [137]. Our exploratory analyses will therefore consider reaction time as another factor that might help mediate the relationship between trait reward sensitivity and psychopathology. In addition, exploratory analyses will also assess whether the associations between substance use, reward sensitivity, and neural measures of reward are stronger for anticipation responses than consumption responses.

\section{Social doors task}

Rationale. Substance use is often tied to social interactions (e.g., binge drinking with friends), and negative social events (e.g., romantic rejection) can trigger relapse in individuals with substance use disorders [50]. 
Although social factors can play a critical role in substance use [20,69], little is known about the relations between substance use, reward sensitivity, and striatal responses to social reward. More specifically, it remains unclear whether substance use is associated with striatal response to social reward, independent of self-reported reward sensitivity. However, striatal response to reward is most often probed in a monetary domain. The few studies to compare brain response to social and monetary reward have results that are difficult to compare due to different task structures or timing. We addressed this challenge by developing a novel fMRI-based variant of our social reward task (Figure 3) [65] that is matched on trial structure, timing, and feedback stimuli with a well-established monetary reward task [138].

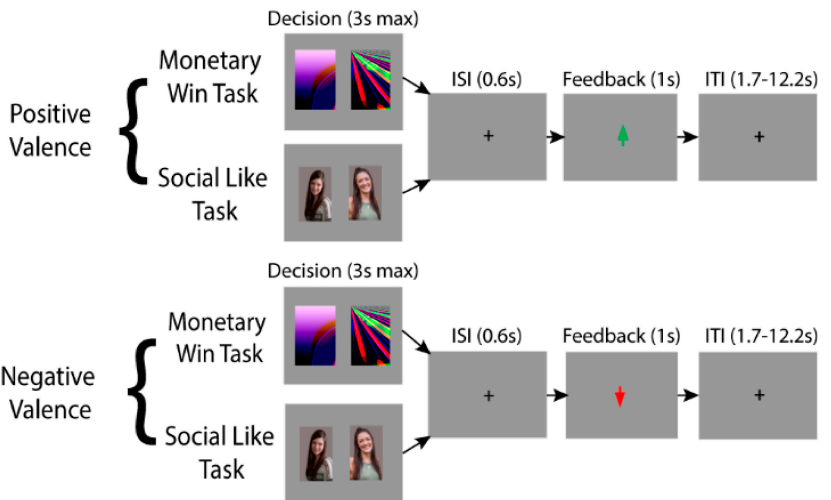

Figure 3. Measuring the Effect of Social Reward Using the Social Doors Task. To measure the effect of social reward consumption, we will use the social doors task. This task requires the participant to guess whether another person likes them or not. The second variant consists of guessing whether an image will have a prize behind it or not. Half of the trials are positive valence, or cases where the subject won money or received social approval and are represented as a green arrow in the outcome phase. The negative valence trials are represented with a red arrow.

Experimental Design. Participants are told that they are completing a social evaluation study and asked to provide a photo of themselves that is purportedly shared with age-matched peers across the country. Purported peers receive a text message asking them to view the photo and indicate whether they would "like” (e.g., social acceptance) or “dislike” (e.g., social rejection) the participant. During fMRI, participants are shown two photos of gender-matched peers at a time and are instructed to guess which of the two peers "liked" them. It is implied that one out of the two peers on the screen is someone who "liked" them, and the second peer is someone who "disliked" them. The monetary guessing task (order counterbalanced) is matched on all features of the social task except that participants choose between two computer generated images of fractals, one containing a monetary reward (50 cents) or a monetary loss (25 cents). The task will consist of 2 runs, each with 60 trials. Our preliminary data show that, on average, the striatum responds similarly to social and nonsocial rewards. However, individual differences in this striatal response emerge in relation to symptoms of anxiety and depression, which are common in the 
context of substance use. A direct comparison between neural response to social and nonsocial reward will be tested in relation to substance use and self-reported reward sensitivity.

Hypotheses. Individuals reporting higher levels of substance use will show exaggerated ventral striatal responses to social rewards relative to monetary rewards, independent of self-reported reward sensitivity. This hypothesis will be evaluated with double subtractions: (Social Acceptance > Social Rejection) > (Monetary Gain > Monetary Loss), where group-level effects are modeled with covariates for substance use and reward sensitivity. Additionally, elevated ventral striatal responses to social reward, relative to monetary reward will be associated with enhanced connectivity with regions modulated by social information (e.g., ventromedial/medial prefrontal cortex to ventral striatum), independent of self-reported reward sensitivity.

Limitation and Future Directions. Although our Social Reward Task permits a direct comparison between social and nonsocial reward and features aspects of social interactions (e.g., peer acceptance/rejection), it may not capture the nuances of real-life social interactions, which involve learning and repeated interactions over time. These processes may be important for understanding how social reward processing contributes to individual differences in substance use. We also note that responses in TPJ during social interactions may be tied to attention [139]. Although recent work has extended and clarified this interpretation [140-142] studies with more nuanced tasks will be necessary to disambiguate social and attentional processes.

\section{Shared reward task}

Rationale. Substance use often occurs in social settings (e.g., binge drinking with friends) or as a result of negative social experiences (e.g., loss of peer relationships). Our group previously has demonstrated that the ventral striatal response to reward is sensitive to social context, with enhanced activation observed when people share a rewarding experience with a close friend relative to a stranger. However, while social factors can play a critical role in substance use [20,69], it remains unclear whether substance use is associated with the striatal response to shared rewarding experiences, independent of self-reported reward sensitivity.

Experimental Design. The goal and structure of this task is designed to examine how experiencing reward or punishment with a partner is affected by social context (Figure 4) [57,62], We will use three partners: a computer, an age- and gender-matched confederate, and a consenting close friend of the participant, represented in the experiment by their picture. After the task, participants will provide subjective ratings of their experiences winning and losing money with each partner. The task will consist of 2 runs, each with 9 blocks containing 8 trials (i.e., 72 trials per run). This design was adapted from previous work [15,57,62] in consultation with Consultant Fareri. 


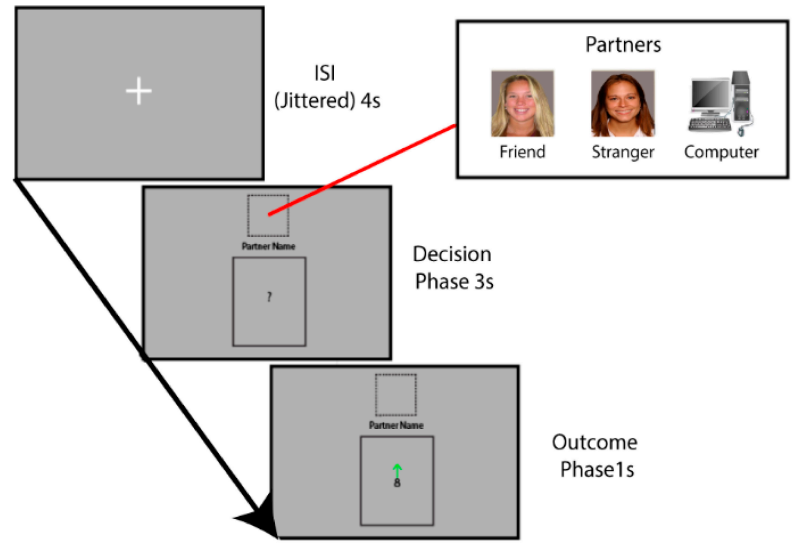

Figure 4. Measuring the Effect of Social Context Using the Shared Reward Task. To measure the effect of social context, we use the shared reward task. This task consists of a card guessing game, with cards containing numbers from 1-9. During the Decision Phase, the participant will learn who will be their partner; a friend, stranger or computer. The subject must guess whether the subsequent card will be greater or less than 5. During the Outcome Phase, the card will be revealed, with a green arrow indicating a correct guess and a red arrow indicating a wrong guess.

Primary Hypotheses. (H2.1) Individuals reporting the highest levels of substance use will show exaggerated striatal responses to the social context of a shared reward, independent of self-reported reward sensitivity. This hypothesis will be evaluated with double subtractions (Friend Reward > Friend Punishment) > (Confederate Reward > Confederate Punishment); Elevated striatal responses to rewards shared with friends relative to strangers will be associated with enhanced connectivity with regions modulated by social information (e.g., temporalparietal junction). This hypothesis also will be examined with double subtractions (cf. H2.1) but using the relevant PPI regressors as inputs to the contrast vector.

Limitations and Future Directions. Although the post-scan ratings provide insight into how participants subjectively experience sharing reward and punishment with each partner [62], the nature of the task (i.e., guessing) does not permit meaningful analyses of trial-to-trial changes in behavior during the imaging session. Future work could extend this paradigm and examine trial-to-trial changes in social learning [143] or trust [57]. In addition, much like our Social Doors Task (Section Social doors task), we acknowledge that TPJ responses to social stimuli could be confounded with attention.

\section{Ultimatum game task}

Rationale. What allows certain people to perform better in bargaining situations than others? It remains unclear how the neural correlates related to executive function and reward sensitivity affect bargaining behavior. Substance use can lead to profound changes in these neural systems, which may lead to maladaptive decision-making. Understanding 
bargaining behavior can inform the psychological and physiological processes that become dysregulated as a result of addiction.

Experimental Design. We will examine bargaining behavior using the Ultimatum (Figure 5) [144] and Dictator Games (Figure 5) [145] ( 15 min, counterbalanced across participants). In the Dictator Game (DG), the participant decides how much of an endowed sum to share with their counterpart. In the Ultimatum Game (UG), the participant chooses a split; however, they also are aware that their counterpart may reject their offer. If their offer is rejected, neither the participant nor their counterpart earns any money for that trial. Finally, the participant acts as a recipient in the UG where they can accept or reject offers. We characterize participants who switch their behavior between the two tasks as acting most strategically to maximize their earnings. The task will consist of 2 runs, each with 36 trials.

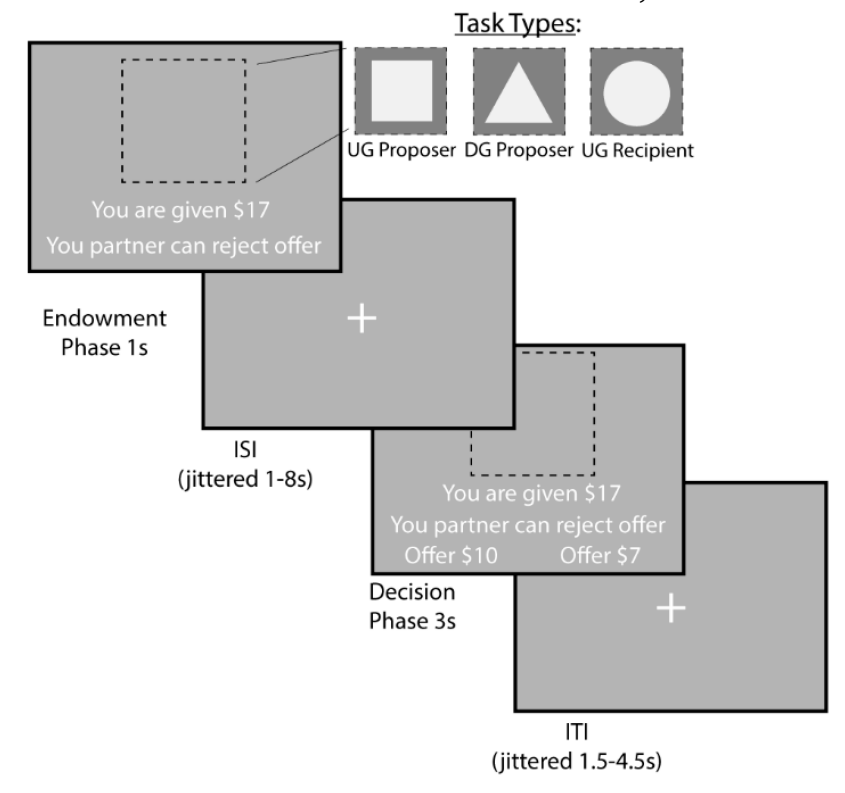

Figure 5. Measuring Strategic Behavior Using the Dictator and Ultimatum Games. To measure strategic behavior, we use the Dictator and Ultimatum games. During the Endowment phase, the participant learns how much money they are given and which task they will do. A square indicates that the participant will be acting as the Proposer in the Ultimatum Game, or deciding how much money to split with a counterpart. A triangle indicates that the participant will act as the Proposer in the Dictator Game. Finally, a circle indicates that the participant will be the Recipient in the Ultimatum Game, which allows them to decide whether they will accept or reject an offer given to them. During the Decision Phase, the participant as a proposer decides to offer More or Less to their counterpart. As a recipient, whether to accept or reject the offer.

On each trial, the participant will be endowed with a sum of money between $\$ 15-\$ 25$. This will be followed by an indication of the type of trial the participant is playing through a target stimulus. If they are acting as the proposer in the DG, they will be presented with a triangle. If they are acting as a proposer in the UG, they will be presented with a square. Finally, if they are acting as a recipient in the UG they will be presented with a circle. During the decision phase as proposer, participants are 
presented with the option to select a More or Less split. During the decision phase as a recipient, participants have the choice whether to accept or reject the offer.

Primary Hypotheses. Bargaining behavior is related to strategic thinking. 1) Individuals scoring higher on the Emotional Intelligence (EI) TEIQe [99] scale will make lower offers as a proposer in the DG, higher offers as a proposer in the UG, and accept more frequently as a recipient in the UG (H3.2): During the endowment phase of the trial, we expect that the response in the ventral striatum and ventromedial prefrontal cortex (vmPFC) will positively vary based on the endowment. (H3.3) There will be differences in neural activation during the decision as a Proposer in the UG and DG tasks in the following regions: dorsolateral prefrontal cortex (dlPFC) as defined by the middle frontal gyrus in the Harvard-Oxford atlas, anterior cingulate cortex, superior parietal lobule, ventromedial prefrontal cortex (vmPFC), and temporoparietal junction (TPJ).

We will compare the differences in activation between UG-Proposer decisions (UG-P) and DG-Proposer (DG-P) decisions, and expect greater dlPFC activation with lower offers in DG-P versus UG-P. Individual differences in strategic bargaining behavior will be associated with choice related responses in the ROIs noted in H2. (H3.4): During the decision phase for the propose conditions, we expect to find that elevated ventral striatal responses to strategic behavior (UG-P > DG-P) will be associated with enhanced effective connectivity with regions modulated by social information (e.g., ventromedial/medial prefrontal cortex to ventral striatum), In addition, these neural effects will be enhanced in individuals with higher levels of self-reported reward sensitivity (i.e., individuals with higher BAS/SR scores will have higher activation and connectivity).

Limitations and Future Directions. Our secondary analyses will group participants into low, medium and high levels of substance use. We will conduct whole brain analyses to examine the effect of social context on neural response to both shared rewards and shared losses as a function of reward sensitivity and substance use. This strategy may provide insight into whether increased substance use also is associated with diminished strategic behavior. Although the UG and DG tasks are examples of bargaining behavior, they may not capture all aspects of real-life bargaining situations, especially in repeated situations. As such, it remains an open question to what extent such tasks generalize to behavior outside the laboratory.

\section{SUMMARY, LIMITATIONS, AND LONG-TERM OUTLOOK}

The relation between reward sensitivity and substance use is poorly understood. The pilot grant described in this Grant Report is the first step in a research program that aims to explore mechanisms that may mediate or moderate the relationship between reward sensitivity and substance use. Although the findings generated by our pilot grant will partly depend on how COVID impacts our project, we hope to make progress toward 
quantifying how reward-dependent corticostriatal connectivity, striatal responses to social reward, and social context relate to substance use. We expect that social context and responses to social reward may elucidate other contributing factors to substance use behavior. We also predict both hyposensitivity and hypersensitivity to reward will have a stronger relationship with substance use than moderate reward sensitivity. Nevertheless, there are several limitations to this work. Given that BIS has also been linked to substance use [46], we will examine its contributions to our primary results in our exploratory analyses. Further, since polysubstance dependence is associated with alterations executive functioning such as working memory and response inhibition [146] we plan to control for variability associated with comorbidity across multiple drug types in tasks that measure social behaviors. Finally, since nicotine can affect reward processing [147,148], we plan to add other relevant measures of substance use to test whether participants are under such influence prior to scanning. Nonetheless, we hypothesize that striatal responses to social and nonsocial reward, and reward-dependent corticostriatal connectivity may serve as an early warning sign that an atrisk person may develop a substance use disorder in the future. For example, neural activation, particularly in the ventral striatum can predict individual choice [149,150].

By exploring how reward sensitivity affects substance use, it may become possible to use neural forecasting techniques to predict how likely it is that a person may relapse in the future. Although this specific question is beyond the scope of the current project, longitudinal research that builds off this project will enable us to follow up with participants to assess whether baseline striatal responses to social and nonsocial reward predict subsequent substance use. Previous research indicates that blunted ventral striatal activity to anticipated rewards predicts drug use in novelty seeking adolescents [30] and the ventral striatum is a significant neural marker of subsequent relapse [151]. However, it remains unclear how responses to social context and social reward are related with substance use. While this study makes early inroads into assessing these relationships, promising results could help identify measures that can predict future substance use in at-risk populations and explore the underlying neural mechanisms associated with substance use. Further, assessing the relation between social reward, social context and substance use can inform how an understudied, but possibly major etiological factor of SUD. Finally, in combining the neural underpinnings of these constructs, this research can inform the psychological and physiological relations underlying substance use. This may affect future theoretical and experimental work in addiction research, informing what makes one atrisk individual more likely to develop substance use disorder compared to another at-risk individual. Ultimately, this research may contribute to interventions and therapies for SUD. 
If we can predict what kind of neural dysfunction is associated with substance use, it may be possible to stimulate those regions to attenuate SUD or risk for developing SUD. Brain stimulation methods have promise for clinical interventions for substance use, such as through transcranial electric stimulation (TES) or transcranial magnetic stimulation (TMS). TMS applied to left frontal pole may dampen frontal and striatal reactivity in chronic alcohol and chronic cocaine users to drug cues [152]. In addition, stimulating the superior frontal gyrus has been associated with modulating cravings for nicotine [153]. Exploring regions associated with substance use and their interconnectivity would help lay the foundation needed to develop stimulation techniques that may minimize cravings and withdrawal. Although current research has investigated striatal responses to nonsocial rewards and its association with SUD, we believe that trait reward sensitivity, social reward processing, and their respective association with neural activation are understudied elements of SUD. A deeper understanding of these underlying mechanisms may inform future clinical interventions.

Current methods that explore the mechanisms that promote risk and resiliency for substance use neglect to consider how a changing environment affects behavior. For example, does the prospect of substances becoming more or less available in the future affect the likelihood of relapse? Among future directions for the research program is to assess how people respond to an evolving environment. How do individuals shift their behavior as their situation gets better or worse over time? How do these factors relate back to classic individual differences that affect behavior, such as risk, losses, uncertainty and ambiguity? In dynamic situations, these factors may affect judgment and decisionmaking to a greater or lesser degree compared to static gambles and lotteries in which they are normally studied. We believe that understanding nuances in decision making in evolving environments over time may provide new insight into pathophysiology in addiction and relapse.

\section{CONFLICTS OF INTEREST}

The authors declare that they have no conflicts of interest.

\section{FUNDING}

This research was funded, in part, by the National Institutes of Health grants R03-DA046733 (awarded to DVS), R21-HD093912 (awarded to JMJ), and R15-MH122927 (awarded to DSF).

\section{REFERENCES}

1. Substance Abuse and Mental Health Services Administration. Key Substance Use and Mental Health Indicators in the United States: Results from the 2018 National Survey on Drug Use and Health. Rockville (MD, US): Center for 
Behavioral Health Statistics and Quality, Substance Abuse and Mental Health Services Administration; 2019. HHS Publication No. PEP19-5068. NSDUH Series H-54.

2. Diehl MM, Lempert KM, Parr AC, Ballard I, Steele VR, Smith DV. Toward an integrative perspective on the neural mechanisms underlying persistent maladaptive behaviors. Eur J Neurosci. 2018;48(3):1870-83.

3. Yücel M, Lubman DI. Neurocognitive and neuroimaging evidence of behavioural dysregulation in human drug addiction: implications for diagnosis, treatment and prevention. Drug Alcohol Rev. 2007;26(1):33-9.

4. Davis L, Uezato A, Newell JM, Frazier E. Major depression and comorbid substance use disorders. Curr Opin Psychiatry. 2008;21(1):14-18.

5. Baskin-Sommers AR, Foti D. Abnormal reward functioning across substance use disorders and major depressive disorder: Considering reward as a transdiagnostic mechanism. Int J Psychophysiol. 2015;98(2):227-39.

6. Nusslock R, Alloy LB. Reward processing and mood-related symptoms: An RDoC and translational neuroscience perspective. J Affect Disord. 2017;216:3 16.

7. Whitton AE, Treadway MT, Pizzagalli DA. Reward processing dysfunction in major depression, bipolar disorder and schizophrenia. Curr Opin Psychiatry. 2015;28(1):7-12.

8. Dalley JW, Robbins TW. Fractionating impulsivity: neuropsychiatric implications. Nat Rev Neurosci. 2017;18(3):158-71.

9. Buckner RL, Andrews-Hanna JR, Schacter DL. The brain's default network: anatomy, function, and relevance to disease. Ann N Y Acad Sci. 2008;1124:1-38.

10. Lerman C, Gu H, Loughead J, Ruparel K, Yang Y, Stein EA. Large-Scale Brain Network Coupling Predicts Acute Nicotine Abstinence Effects on Craving and Cognitive Function. JAMA Psychiatry. 2014;71(5):523.

11. Liang X, He Y, Salmeron BJ, Gu H, Stein EA, Yang Y. Interactions between the salience and default-mode networks are disrupted in cocaine addiction. J Neurosci Off J Soc Neurosci. 2015;35(21):8081-90.

12. Ma N, Liu Y, Fu X-M, Li N, Wang C-X, Zhang H, et al. Abnormal brain defaultmode network functional connectivity in drug addicts. PLoS One. 2011;6(1):e16560.

13. Sutherland MT, McHugh MJ, Pariyadath V, Stein EA. Resting state functional connectivity in addiction: Lessons learned and a road ahead. NeuroImage. 2012;62(4):2281-95.

14. Chein J, Albert D, O’Brien L, Uckert K, Steinberg L. Peers increase adolescent risk taking by enhancing activity in the brain's reward circuitry. Dev Sci. 2011;14(2):F1-10.

15. Fareri DS, Delgado MR. Social Rewards and Social Networks in the Human Brain. Neurosci Rev J Bringing Neurobiol Neurol Psychiatry. 2014;20(4):387402.

16. Beer JS, John OP, Scabini D, Knight RT. Orbitofrontal cortex and social behavior: integrating self-monitoring and emotion-cognition interactions. J Cogn Neurosci. 2006;18(6):871-9. 
17. Izuma K, Saito D, Sadato N. Processing of the Incentive for Social Approval in the Ventral Striatum during Charitable Donation. J Cogn Neurosci. 2009;22:621-31.

18. Walsh E, Carl H, Eisenlohr-Moul T, Minkel J, Crowther A, Moore T, et al. Attenuation of Frontostriatal Connectivity During Reward Processing Predicts Response to Psychotherapy in Major Depressive Disorder. Neuropsychopharmacology. 2017;42(4):831-43.

19. Hung C-C, Zhang S, Chen C-M, Duann J-R, Lin C-P, Lee TS-H, et al. Striatal functional connectivity in chronic ketamine users: a pilot study. Am J Drug Alcohol Abuse. 2020;46(1):31-43.

20. Heilig M, Epstein DH, Nader MA, Shaham Y. Time to connect: bringing social context into addiction neuroscience. Nat Rev Neurosci. 2016;17(9):592-9.

21. Goldstein RZ, Volkow ND. Dysfunction of the prefrontal cortex in addiction: neuroimaging findings and clinical implications. Nat Rev Neurosci. 2011;12(11):652-69.

22. Koob GF, Le Moal M. Drug addiction, dysregulation of reward, and allostasis. Neuropsychopharmacol Off Publ Am Coll Neuropsychopharmacol. 2001;24(2):97-129.

23. Koob GF. Addiction is a Reward Deficit and Stress Surfeit Disorder. Front Psychiatry. 2013;4:72.

24. Volkow ND, Wang G-J, Fowler JS, Tomasi D, Telang F, Baler R. Addiction: decreased reward sensitivity and increased expectation sensitivity conspire to overwhelm the brain's control circuit. BioEssays News Rev Mol Cell Dev Biol. 2010 Sep;32(9):748-55.

25. Blum K, Braverman ER, Holder JM, Lubar JF, Monastra VJ, Miller D, et al. Reward deficiency syndrome: a biogenetic model for the diagnosis and treatment of impulsive, addictive, and compulsive behaviors. J Psychoactive Drugs. 2000;32(Suppl):i-iv, 1-112.

26. Koob GF, Volkow ND. Neurocircuitry of Addiction. Neuropsychopharmacology. 2010;35(1):217-38.

27. Ersche KD, Roiser JP, Abbott S, Craig KJ, Müller U, Suckling J, et al. Response perseveration in stimulant dependence is associated with striatal dysfunction and can be ameliorated by a $\mathrm{D}(2 / 3)$ receptor agonist. Biol Psychiatry. 2011;70(8):754-62.

28. Meyer PJ, King CP, Ferrario CR. Motivational Processes Underlying Substance Abuse Disorder. Curr Top Behav Neurosci. 2016;27:473-506.

29. Luijten M, Schellekens AF, Kühn S, Machielse MWJ, Sescousse G. Disruption of Reward Processing in Addiction: An Image-Based Meta-analysis of Functional Magnetic Resonance Imaging Studies. JAMA Psychiatry. 2017;74(4):387-98.

30. Büchel C, Peters J, Banaschewski T, Bokde ALW, Bromberg U, Conrod PJ, et al. Blunted ventral striatal responses to anticipated rewards foreshadow problematic drug use in novelty-seeking adolescents. Nat Commun. 2017;8:14140.

31. Joyner KJ, Bowyer CB, Yancey JR, Venables NC, Foell J, Worthy DA, et al. Blunted Reward Sensitivity and Trait Disinhibition Interact to Predict 
Substance Use Problems. Clin Psychol Sci J Assoc Psychol Sci. 2019;7(5):110924.

32. Quello SB, Brady KT, Sonne SC. Mood Disorders and Substance Use Disorder: A Complex Comorbidity. Sci Pract Perspect. 2005;3(1):13-21.

33. Furman DJ, Hamilton JP, Gotlib IH. Frontostriatal functional connectivity in major depressive disorder. Biol Mood Anxiety Disord. 2011;1:11.

34. Hao ZY, Zhong Y, Ma ZJ, Xu HZ, Kong JY, Wu Z, et al. Abnormal resting-state functional connectivity of hippocampal subfields in patients with major depressive disorder. BMC Psychiatry. 2020;20(1):71.

35. Ng TH, Alloy LB, Smith DV. Meta-analysis of reward processing in major depressive disorder reveals distinct abnormalities within the reward circuit. Transl Psychiatry. 2019;9(1):1-10.

36. Kumar P, Goer F, Murray L, Dillon DG, Beltzer ML, Cohen AL, et al. Impaired reward prediction error encoding and striatal-midbrain connectivity in depression. Neuropsychopharmacol Off Publ Am Coll Neuropsychopharmacol. 2018;43(7):1581-8.

37. Liu W, Roiser JP, Wang L, Zhu Y, Huang J, Neumann DL, et al. Anhedonia is associated with blunted reward sensitivity in first-degree relatives of patients with major depression. J Affect Disord. 2016;190:640-8.

38. Gray JA. The psychophysiological basis of introversion-extraversion. Behav Res Ther. 1970;8(3):249-66.

39. Carver C, White T. Behavioral Inhibition, Behavioral Activation, and Affective Responses to Impending Reward and Punishment: The BIS/BAS Scales. J Pers Soc Psychol. 1994;67:319-33.

40. Torrubia R, Avila C, Moltó J, Caseras X. The Sensitivity to Punishment and Sensitivity to Reward Questionnaire (SPSRQ) as a measure of Gray's Anxiety and Impulsivity dimensions. Personal Individ Differ. 2001;31:837-62.

41. Alloy LB, Abramson LY, Walshaw PD, Gerstein RK, Keyser JD, Whitehouse WG, et al. Behavioral approach system (BAS)-relevant cognitive styles and bipolar spectrum disorders: Concurrent and prospective associations. J Abnorm Psychol. 2009;118(3):459-71.

42. Martin LE, Potts GF. Reward sensitivity in impulsivity. Neuroreport. 2004;15(9):1519-22.

43. Costumero V, Barrós-Loscertales A, Bustamante JC, Ventura-Campos N, Fuentes P, Rosell-Negre P, et al. Reward Sensitivity Is Associated with Brain Activity during Erotic Stimulus Processing. PLoS One. 2013;8(6):e66940.

44. Costumero V, Barrós-Loscertales A, Fuentes P, Rosell-Negre P, Bustamante JC, Ávila C. BAS-drive trait modulates dorsomedial striatum activity during reward response-outcome associations. Brain Imaging Behav. 2016;10(3):86979.

45. Yamamoto DJ, Banich MT, Regner MF, Sakai JT, Tanabe J. Behavioral approach and orbitofrontal cortical activity during decision-making in substance dependence. Drug Alcohol Depend. 2017;180:234-40.

46. Franken IHA, Muris P. BIS/BAS personality characteristics and college students’ substance use. Personal Individ Differ. 2006;40(7):1497-503. 
47. Balconi M, Finocchiaro R. Decisional impairments in cocaine addiction, reward bias, and cortical oscillation "unbalance.” Neuropsychiatr Dis Treat. 2015;11:777-86.

48. Lawn W, Freeman TP, Hindocha C, Mokrysz C, Das RK, Morgan CJA, et al. The effects of nicotine dependence and acute abstinence on the processing of drug and non-drug rewards. Psychopharmacology (Berl). 2015;232(14):2503-17.

49. Genovese JEC, Wallace D. Reward sensitivity and substance abuse in middle school and high school students. J Genet Psychol Res Theory Hum Dev. 2007;168(4):465-9.

50. Marlatt GA, Donovan DM. Relapse prevention: Maintenance strategies in the treatment of addictive behaviors. 2nd ed. New York (NY, US): The Guilford Press; 2005. p. xiv, 416.

51. Oh H, Lee J, Gosnell SN, Patriquin M, Kosten T, Salas R. Orbitofrontal, dorsal striatum, and habenula functional connectivity in psychiatric patients with substance use problems. Addict Behav. 2020;108:106457.

52. Tricomi E, Rangel A, Camerer CF, O’Doherty JP. Neural evidence for inequality-averse social preferences. Nature. 2010;463(7284):1089-91.

53. Bault N, Joffily M, Rustichini A, Coricelli G. Medial prefrontal cortex and striatum mediate the influence of social comparison on the decision process. Proc Natl Acad Sci. 2011;108(38):16044-9.

54. Fliessbach K, Weber B, Trautner P, Dohmen T, Sunde U, Elger C, et al. Social Comparison Affects Reward-Related Brain Activity in the Human Ventral Striatum. Science. 2007 Dec 1;318:1305-8.

55. Báez-Mendoza R, Schultz W. The role of the striatum in social behavior. Front Neurosci. 2013;7:233.

56. Tamir DI, Mitchell JP. Disclosing information about the self is intrinsically rewarding. Proc Natl Acad Sci U S A. 2012;109(21):8038-43.

57. Fareri DS, Chang LJ, Delgado MR. Computational Substrates of Social Value in Interpersonal Collaboration. J Neurosci. 2015;35(21):8170-80.

58. Simon D, Becker MPI, Mothes-Lasch M, Miltner WHR, Straube T. Effects of social context on feedback-related activity in the human ventral striatum. NeuroImage. 2014;99:1-6.

59. Behrens TEJ, Hunt LT, Rushworth MFS. The computation of social behavior. Science. 2009;324(5931):1160-4.

60. Saxe R. Uniquely human social cognition. Curr Opin Neurobiol. 2006;16(2):235-9.

61. Mars RB, Neubert F-X, Noonan MP, Sallet J, Toni I, Rushworth MFS. On the relationship between the "default mode network" and the "social brain". Front Hum Neurosci. 2012;6:189.

62. Fareri D, Niznikiewicz M, Lee V, Delgado M. Social Network Modulation of Reward-Related Signals. J Neurosci Off J Soc Neurosci. 2012;32:9045-52.

63. Smith AR, Steinberg L, Strang N, Chein J. Age differences in the impact of peers on adolescents' and adults' neural response to reward. Dev Cogn Neurosci. 2015;11:75-82. 
64. O’Brien L, Albert D, Chein J, Steinberg L. Adolescents Prefer More Immediate Rewards When in the Presence of their Peers. J Res Adolesc. 2011;21(4):74753.

65. Distefano A, Jackson F, Levinson AR, Infantolino ZP, Jarcho JM, Nelson BD. A comparison of the electrocortical response to monetary and social reward. Soc Cogn Affect Neurosci. 2018;13(3):247-55.

66. Wake SJ, Izuma K. A common neural code for social and monetary rewards in the human striatum. Soc Cogn Affect Neurosci. 2017;12(10):1558-64.

67. Smith DV, Clithero JA, Boltuck SE, Huettel SA. Functional connectivity with ventromedial prefrontal cortex reflects subjective value for social rewards. Soc Cogn Affect Neurosci. 2014;9(12):2017-25.

68. van den Bos W, Vahl P, Güroğlu B, van Nunspeet F, Colins O, Markus M, et al. Neural correlates of social decision-making in severely antisocial adolescents. Soc Cogn Affect Neurosci. 2014;9(12):2059-66.

69. Volkow ND, Baler RD, Goldstein RZ. Addiction: Pulling at the Neural Threads of Social Behaviors. Neuron. 2011;69(4):599-602.

70. Côté S, Decelles K, Mccarthy J, van Kleef G, Hideg I. The Jekyll and Hyde of Emotional Intelligence Emotion-Regulation Knowledge Facilitates Both Prosocial and Interpersonally Deviant Behavior. Psychol Sci. 2011;22:1073-80.

71. Nozaki Y, Koyasu M. The Relationship between Trait Emotional Intelligence and Interaction with Ostracized Others' Retaliation. PLoS One. 2013;8(10):e77579.

72. Scheres A, Sanfey A. Individual differences in decision making: Drive and Reward Responsiveness affect strategic bargaining in economic games. Behav Brain Funct. 2006;2:35.

73. De Neys W, Novitskiy N, Geeraerts L, Ramautar J, Wagemans J. Cognitive Control and Individual Differences in Economic Ultimatum Decision-Making. PLoS One. 2011;6(11):e27107.

74. Spitzer M, Fischbacher U, Herrnberger B, Grön G, Fehr E. The neural signature of social norm compliance. Neuron. 2007;56(1):185-96.

75. Delgado MR, Nystrom LE, Fissell C, Noll DC, Fiez JA. Tracking the hemodynamic responses to reward and punishment in the striatum. J Neurophysiol. 2000;84(6):3072-7.

76. Knutson B, Westdorp A, Kaiser E, Hommer D. FMRI visualization of brain activity during a monetary incentive delay task. NeuroImage. 2000;12(1):207.

77. Quarmley ME, Nelson BD, Clarkson T, White LK, Jarcho JM. I Knew You Weren't Going to Like Me! Neural Response to Accurately Predicting Rejection Is Associated With Anxiety and Depression. Front Behav Neurosci. 2019;13:219.

78. Smith DV, Gseir M, Speer ME, Delgado MR. Toward a cumulative science of functional integration: A meta-analysis of psychophysiological interactions. Hum Brain Mapp. 2016;37(8):2904-17.

79. Smith DV, Rigney AE, Delgado MR. Distinct Reward Properties are Encoded via Corticostriatal Interactions. Sci Rep. 2016;6(1):1-12. 
80. McCloskey MS, Berman ME. Alcohol intoxication and self-aggressive behavior. J Abnorm Psychol. 2003;112(2):306-11.

81. McNutt MK, Bradford M, Drazen JM, Hanson B, Howard B, Jamieson KH, et al. Transparency in authors' contributions and responsibilities to promote integrity in scientific publication. Proc Natl Acad Sci. 2018;115(11):2557-60.

82. Strange K. Authorship: why not just toss a coin? Am J Physiol Cell Physiol. 2008;295(3):C567-75.

83. Gorgolewski KJ, Varoquaux G, Rivera G, Schwarz Y, Ghosh SS, Maumet C, et al. NeuroVault.org: a web-based repository for collecting and sharing unthresholded statistical maps of the human brain. Front Neuroinformatics. 2015;9:8.

84. Alloy LB, Abramson LY, Walshaw PD, Cogswell A, Smith JM, Neeren AM, et al. Behavioral Approach System (BAS) Sensitivity and Bipolar Spectrum Disorders: A Retrospective and Concurrent Behavioral High-Risk Design. Motiv Emot. 2006;30(2):143-55.

85. Alloy LB, Bender RE, Whitehouse WG, Wagner CA, Liu RT, Grant DA, et al. High Behavioral Approach System (BAS) sensitivity, reward responsiveness, and goal-striving predict first onset of bipolar spectrum disorders: A prospective behavioral high-risk design. J Abnorm Psychol. 2012;121(2):33951.

86. Liu RT, Burke TA, Abramson LY, Alloy LB. The Behavioral Approach System (BAS) Model of Vulnerability to Bipolar Disorder: Evidence of a Continuum in BAS Sensitivity across Adolescence. J Abnorm Child Psychol. 2018;46(6):133349.

87. Spitzer RL, Endicott J, Robins E. Research diagnostic criteria: rationale and reliability. Arch Gen Psychiatry. 1978;35(6):773-82.

88. First MB. Structured Clinical Interview for the DSM (SCID). In: The Encyclopedia of Clinical Psychology. Atlanta (US): American Cancer Society; 2015; p. 1-6.

89. Beck AT, Steer RA, Ball R, Ranieri W. Comparison of Beck Depression Inventories -IA and -II in psychiatric outpatients. J Pers Assess. 1996;67(3):58897.

90. Youngstrom EA, Murray G, Johnson SL, Findling RL. The 7 Up 7 Down Inventory: A 14-item measure of manic and depressive tendencies carved from the General Behavior Inventory. Psychol Assess. 2013;25(4):1377-83.

91. Altman EG, Hedeker D, Peterson JL, Davis JM. The Altman Self-Rating Mania Scale. Biol Psychiatry. 1997;42(10):948-55.

92. Ehrman RN, Robbins SJ. Reliability and validity of 6-month timeline reports of cocaine and heroin use in a methadone population. J Consult Clin Psychol. 1994;62(4):843-50.

93. Sobell MB, Sobell LC, Klajner F, Pavan D, Basian E. The reliability of a timeline method for assessing normal drinker college students' recent drinking history: utility for alcohol research. Addict Behav. 1986;11(2):149-61.

94. Berman AH, Bergman H, Palmstierna T, Schlyter F. Evaluation of the Drug Use Disorders Identification Test (DUDIT) in criminal justice and detoxification 
settings and in a Swedish population sample. Eur Addict Res. 2005;11(1):2231.

95. Moberg D, Hahn L. The adolescent drug involvement scale. J Child Adolesc Subst Abuse. 1991;2:75-88.

96. Moberg DP. The Adolescent Alcohol and Drug Involvement Scale: An instrument for measuring adolescent use and misuse of alcohol. J Stud Alcohol. 2000;(40):291-300.

97. Baron-Cohen S, Wheelwright S, Skinner R, Martin J, Clubley E. The autismspectrum quotient (AQ): evidence from Asperger syndrome/high-functioning autism, males and females, scientists and mathematicians. J Autism Dev Disord. 2001;31(1):5-17.

98. Davis MH. Measuring individual differences in empathy: Evidence for a multidimensional approach. J Pers Soc Psychol. 1983;44(1):113-26.

99. Petrides KV. Psychometric properties of the Trait Emotional Intelligence Questionnaire (TEIQue). In: Assessing emotional intelligence: Theory, research, and applications. New York (NY, US): Springer Science + Business Media; 2009. p. 85-101.

100. Cooper ML. Motivations for alcohol use among adolescents: Development and validation of a four-factor model. Psychol Assess. 1994;6(2):117-28.

101. Simons J, Correia CJ, Carey KB. A comparison of motives for marijuana and alcohol use among experienced users. Addict Behav. 2000;25(1):153-60.

102. Simons JS, Gaher RM. The Distress Tolerance Scale: Development and Validation of a Self-Report Measure. Motiv Emot. 2005;29(2):83-102.

103. Esteban O, Markiewicz CJ, Blair RW, Moodie CA, Isik AI, Erramuzpe A, et al. fMRIPrep: a robust preprocessing pipeline for functional MRI. Nat Methods. 2019;16(1):111-6.

104. Gorgolewski K, Burns CD, Madison C, Clark D, Halchenko YO, Waskom ML, et al. Nipype: a flexible, lightweight and extensible neuroimaging data processing framework in python. Front Neuroinformatics. 2011;5:13.

105. Gorgolewski KJ, Esteban O, Ellis DG, Notter MP, Ziegler E, Johnson H, et al. Nipype: a flexible, lightweight and extensible neuroimaging data processing framework in Python. 0.13.1. Available from: https://zenodo.org/record/581704. Accessed 2020 Oct 28.

106. Tustison NJ, Avants BB, Cook PA, Zheng Y, Egan A, Yushkevich PA, et al. N4ITK: improved N3 bias correction. IEEE Trans Med Imaging. 2010;29(6):1310-20.

107. Avants BB, Epstein CL, Grossman M, Gee JC. Symmetric diffeomorphic image registration with cross-correlation: Evaluating automated labeling of elderly and neurodegenerative brain. Med Image Anal. 2008;12(1):26-41.

108. Zhang Y, Brady M, Smith S. Segmentation of brain MR images through a hidden Markov random field model and the expectation-maximization algorithm. IEEE Trans Med Imaging. 2001;20(1):45-57.

109. Fonov V, Evans A, McKinstry R, Almli C, Collins D. Unbiased nonlinear average age-appropriate brain templates from birth to adulthood. NeuroImage. 2009;47:S102.

110. Evans AC, Janke AL, Collins DL, Baillet S. Brain templates and atlases. NeuroImage. 2012;62(2):911-22. 
111. Glasser MF, Sotiropoulos SN, Wilson JA, Coalson TS, Fischl B, Andersson JL, et al. The minimal preprocessing pipelines for the Human Connectome Project. NeuroImage. 2013;80:105-24.

112. Jenkinson M, Bannister P, Brady M, Smith S. Improved Optimization for the Robust and Accurate Linear Registration and Motion Correction of Brain Images. NeuroImage. 2002;17(2):825-41.

113. Greve DN, Fischl B. Accurate and robust brain image alignment using boundary-based registration. NeuroImage. 2009;48(1):63-72.

114. Cox RW, Hyde JS. Software tools for analysis and visualization of fMRI data. NMR Biomed. 1997;10(4-5):171-8.

115. Pruim RHR, Mennes M, Buitelaar JK, Beckmann CF. Evaluation of ICA-AROMA and alternative strategies for motion artifact removal in resting state fMRI. NeuroImage. 2015;112:278-87.

116. Power JD, Schlaggar BL, Petersen SE. Recent progress and outstanding issues in motion correction in resting state fMRI. NeuroImage. 2015;0:536-51.

117. Lanczos C. Evaluation of Noisy Data. J Soc Ind Appl Math Ser B Numer Anal. 1964;1(1):76-85.

118. Abraham A, Pedregosa F, Eickenberg M, Gervais P, Mueller A, Kossaifi J, et al. Machine learning for neuroimaging with scikit-learn. Front Neuroinformatics. 2014;8:14.

119. Smith S, Jenkinson M, Woolrich M, Beckmann C, Behrens T, Johansen-Berg H, et al. Advances in functional and structural MR image analysis and implementation as FSL. NeuroImage. 2004;23(Suppl 1):S208-19.

120. Woolrich MW, Ripley BD, Brady M, Smith SM. Temporal autocorrelation in univariate linear modeling of FMRI data. NeuroImage. 2001;14(6):1370-86.

121. Friston KJ, Buechel C, Fink GR, Morris J, Rolls E, Dolan RJ. Psychophysiological and modulatory interactions in neuroimaging. NeuroImage. 1997;6(3):218-29.

122. Friston KJ. Functional and effective connectivity: a review. Brain Connect. 2011;1(1):13-36.

123. Smith DV, Delgado MR. Meta-analysis of psychophysiological interactions: Revisiting cluster-level thresholding and sample sizes. Hum Brain Mapp. 2017;38(1):588-91.

124. Tziortzi AC, Searle GE, Tzimopoulou S, Salinas C, Beaver JD, Jenkinson M, et al. Imaging dopamine receptors in humans with [11C]-(+)-PHNO: Dissection of D3 signal and anatomy. NeuroImage. 2011;54(1):264-77.

125. O'Reilly JX, Woolrich MW, Behrens TEJ, Smith SM, Johansen-Berg H. Tools of the trade: psychophysiological interactions and functional connectivity. Soc Cogn Affect Neurosci. 2012;7(5):604-9.

126. Fareri DS, Smith DV, Delgado MR. The influence of relationship closeness on default-mode network connectivity during social interactions. Soc Cogn Affect Neurosci. 2020;15(3):261-71.

127. Utevsky AV, Smith DV, Young JS, Huettel SA. Large-Scale Network Coupling with the Fusiform Cortex Facilitates Future Social Motivation. eNeuro. 2017;4(5):ENEURO.0084-17.2017. 
128. Nickerson LD, Smith SM, Öngür D, Beckmann CF. Using Dual Regression to Investigate Network Shape and Amplitude in Functional Connectivity Analyses. Front Neurosci. 2017;11:115.

129. Filippini N, MacIntosh BJ, Hough MG, Goodwin GM, Frisoni GB, Smith SM, et al. Distinct patterns of brain activity in young carriers of the APOE- 44 allele. Proc Natl Acad Sci U S A. 2009;106(17):7209-14.

130. Woolrich MW, Behrens TEJ, Beckmann CF, Jenkinson M, Smith SM. Multilevel linear modelling for FMRI group analysis using Bayesian inference. NeuroImage. 2004;21(4):1732-47.

131. Smith DV, Utevsky AV, Bland AR, Clement N, Clithero JA, Harsch AEW, et al. Characterizing Individual Differences in Functional Connectivity Using DualRegression and Seed-Based Approaches. NeuroImage. 2014;95:1-12.

132. Tepfer L, Alloy L, Smith D. Family History of Depression is Associated with Alterations in Task-Dependent Connectivity between the Cerebellum and Ventromedial Prefrontal Cortex. bioRxiv 851477 [Preprint]. 2019 Nov 21. https://doi.org/10.1101/851477

133. Eklund A, Nichols TE, Knutsson H. Cluster failure: Why fMRI inferences for spatial extent have inflated false-positive rates. Proc Natl Acad Sci U S A. 2016 12;113(28):7900-5.

134. Woo C-W, Krishnan A, Wager TD. Cluster-extent based thresholding in fMRI analyses: pitfalls and recommendations. NeuroImage. 2014;91:412-9.

135. Smith SM, Nichols TE. Threshold-free cluster enhancement: addressing problems of smoothing, threshold dependence and localisation in cluster inference. NeuroImage. 2009;44(1):83-98.

136. Knutson B, Fong GW, Adams CM, Varner JL, Hommer D. Dissociation of reward anticipation and outcome with event-related fMRI. NeuroReport. 2001;12(17):3683-7.

137. Clithero JA, Reeck C, Carter RM, Smith DV, Huettel SA. Nucleus Accumbens Mediates Relative Motivation for Rewards in the Absence of Choice. Front Hum Neurosci. 2011;5:87.

138. Proudfit GH. The reward positivity: From basic research on reward to a biomarker for depression. Psychophysiology. 2015;52(4):449-59.

139. Cabeza R, Ciaramelli E, Moscovitch M. Cognitive contributions of the ventral parietal cortex: an integrative theoretical account. Trends Cogn Sci. 2012;16(6):338-52.

140. Carter RM, Huettel SA. A nexus model of the temporal-parietal junction. Trends Cogn Sci. 2013;17(7):328-36.

141. Kahnt T, Tobler PN. Salience Signals in the Right Temporoparietal Junction Facilitate Value-Based Decisions. J Neurosci. 2013;33(3):863-9.

142. Tusche A, Böckler A, Kanske P, Trautwein F-M, Singer T. Decoding the Charitable Brain: Empathy, Perspective Taking, and Attention Shifts Differentially Predict Altruistic Giving. J Neurosci Off J Soc Neurosci. 2016 27;36(17):4719-32.

143. Behrens TEJ, Hunt LT, Woolrich MW, Rushworth MFS. Associative learning of social value. Nature. 2008;456(7219):245-9. 
144. Güth W, Schmittberger R, Schwarze B. An experimental analysis of ultimatum bargaining. J Econ Behav Organ. 1982;3(4):367-88.

145. Kahneman D, Knetsch JL, Thaler RH. Fairness and the Assumptions of Economics. J Bus. 1986;59(S4):S285.

146. Valls-Serrano C, Verdejo-García A, Caracuel A. Planning deficits in polysubstance dependent users: Differential associations with severity of drug use and intelligence. Drug Alcohol Depend. 2016;162:72-8.

147. Perkins KA, Karelitz JL, Boldry MC. Nicotine Acutely Enhances Reinforcement from Non-Drug Rewards in Humans. Front Psychiatry. 2017;8:65.

148. Xu X, Clark US, David SP, Mulligan RC, Knopik VS, McGeary J, et al. Effects of Nicotine Deprivation and Replacement on BOLD-fMRI Response to Smoking Cues as a Function of DRD4 VNTR Genotype. Nicotine Tob Res. 2014;16(7):93947.

149. Genevsky A, Knutson B. Neural Affective Mechanisms Predict Market-Level Microlending. Psychol Sci. 2015;26(9):1411-22.

150. Knutson B, Genevsky A. Neuroforecasting Aggregate Choice. Curr Dir Psychol Sci. 2018;27(2):110-5. doi: 10.1177/0963721417737877

151. MacNiven KH, Jensen ELS, Borg N, Padula CB, Humphreys K, Knutson B. Association of Neural Responses to Drug Cues With Subsequent Relapse to Stimulant Use. JAMA Netw Open. 2018;1(8): e186466.

152. Kearney-Ramos TE, Dowdle LT, Lench DH, Mithoefer OJ, Devries WH, George MS, et al. Transdiagnostic Effects of Ventromedial Prefrontal Cortex Transcranial Magnetic Stimulation on Cue Reactivity. Biol Psychiatry Cogn Neurosci Neuroimaging. 2018;3(7):599-609.

153. Rose JE, McClernon FJ, Froeliger B, Behm FM, Preud'homme X, Krystal AD. Repetitive transcraniamagnetic stimulation of the superior frontal gyrus modulates craving for cigarettes. Biol Psychiatry. 2011;70(8):794-9.

How to cite this article:

Sazhin D, Frazier AM, Haynes CR, Johnston CR, Chat IKY, Dennison JB, et al. The Role of Social Reward and Corticostriatal Connectivity in Substance Use. J Psychiatry Brain Sci. 2020;5:e200024. https://doi.org/10.20900/jpbs.20200024 